\title{
BETs that cover the spread from acquired to heritable heart failure
}

\author{
Michael Alexanian ${ }^{1}$ and Saptarsi M. Haldar $r^{1,2,3}$ \\ 'Cladstone Institutes, San Francisco, California, USA. ${ }^{2}$ Division of Cardiology, Department of Medicine, UCSF, San Francisco, California, USA. ${ }^{3}$ Amgen Research, South San Francisco, California, USA.
}

\begin{abstract}
Heart failure (HF) with reduced contractile function is a common and lethal syndrome in which the heart cannot pump blood to adequately meet bodily demands, resulting in high mortality despite the current standard of care. In modern societies, the most common drivers of HF are ischemic heart disease and hypertension. However, in a substantial subset of cases, patients present with dilated and poorly contracting hearts without evidence of common inciting stressors, a syndrome called dilated cardiomyopathy (DCM). Genome sequencing has identified a host of deleterious germline variants in key cardiomyocyte genes as causes of heritable DCM, including mutations in LMNA, which encodes the nuclear lamina-associated protein lamin $A / C$. In this issue of the $J C I$, Auguste et al. generate a mouse model of DCM in which they delete Lmna in cardiomyocytes and discover that bromodomain and extraterminal (BET) protein activation is a druggable epigenetic mechanism of disease pathogenesis in this heritable HF syndrome.
\end{abstract}

\section{Laminopathies}

LMNA encodes an intermediate filament protein with several complex functions, including maintenance of nuclear ultrastructure, mechanotransduction, and regulation of gene expression (1). Autosomal dominant germline mutations in LMNA cause a heterogenous group of multisystem disorders called laminopathies, whose manifestations include cardiomyopathy, muscular dystrophy, metabolic disturbances, and accelerated aging (progeria) (1). Several LMNA mutations have a strong predilection to cause dilated cardiomyopathy (DCM), cardiac conduction abnormalities, and sudden cardiac death $(1,2)$. Currently, there are no specific disease-modifying therapies for laminopathies, catalyzing interest in the development of preclinical models for drug discovery and gene therapy.
Although several groups have developed mouse models harboring pathogenic germline Lmna mutations, the cardiomyocyte-specific role of LMNA in vivo is poorly understood. In this issue of the JCI, Auguste et al. deleted Lmna in a cardiomyocytespecific manner in mice (Lmna-cKO) using the Myh6-Cre line, which principally drives gene deletion during postnatal cardiac maturation (3). The researchers found that Lmna-cKO mice had $100 \%$ mortality by 4 weeks of age, preceded by severe cardiac dilation, contractile dysfunction, fibrosis, and cardiac rhythm disturbances that began after 2 weeks of age. Lmna haploinsufficiency caused a milder form of DCM, suggesting dosage sensitivity (3). These phenotypes are observed in patients with LMNA-associated DCM, suggesting cardiomyocyte-specific Lmna loss can trigger major cardiac manifestations of laminopathy.

Related Article: p. 4740

Conflict of interest: SMH is an executive, officer, and shareholder of Amgen, Inc. and is a scientific cofounder and shareholder of Tenaya Therapeutics.

Copyright: (c) 2020, American Society for Clinical Investigation.

Reference information: J Clin Invest. 2020;130(9):4536-4539. https://doi.org/10.1172/JCI140304.

To better understand the molecular pathways dysregulated by Lmna deletion, the team performed bulk RNA sequencing (RNA-Seq) of cardiomyocytes isolated from homozygous Lmna-cKO mice. To detect early effects of Lmna deletion and avoid secondary transcriptomic effects of advanced heart failure (HF), they profiled cardiomyocytes from two-week-old mice, a time point preceding onset of cardiac dysfunction. Analyses of differentially expressed genes revealed upregulation of profibrotic programs and predicted activation of specific signaling pathways, including activation of the bromodomain and extraterminal (BET) family of chromatin regulatory proteins $(3-5)$. BETs are a highly conserved family of epigenetic "reader" proteins consisting of the ubiquitously expressed BRD2, BRD3, and BRD4 and the testis-specific BRDT. BET proteins bind lysine-acetylated histones and transcription factors via two N-terminal acetyl-lysine recognition domains, or bromodomains. Once recruited to hyperacetylated regions of chromatin, BET proteins scaffold macromolecular complexes that coactivate gene transcription (5). Consistent with this mechanism, chromatin immunoprecipitation with DNA sequencing (ChIP-Seq) for BRD4 in primary isolated cardiomyocytes from control versus Lmna-cKO mice revealed preferential BRD4 recruitment near genes that were upregulated in mutant cardiomyocytes, confirming increased BRD4 function in the setting of Lmna deficiency (3).

\section{BET proteins are druggable regulators of transcription}

These observations are particularly intriguing because BET proteins are druggable regulators of transcription. Potent and specific pan-BET-inhibitory small-molecule probes such as JQ1 (4) and iBET (6) were developed in 2010 and have been shown to block growth of several tumor types. This class of small molecules, called BET bromodomain inhibitors, spe- 

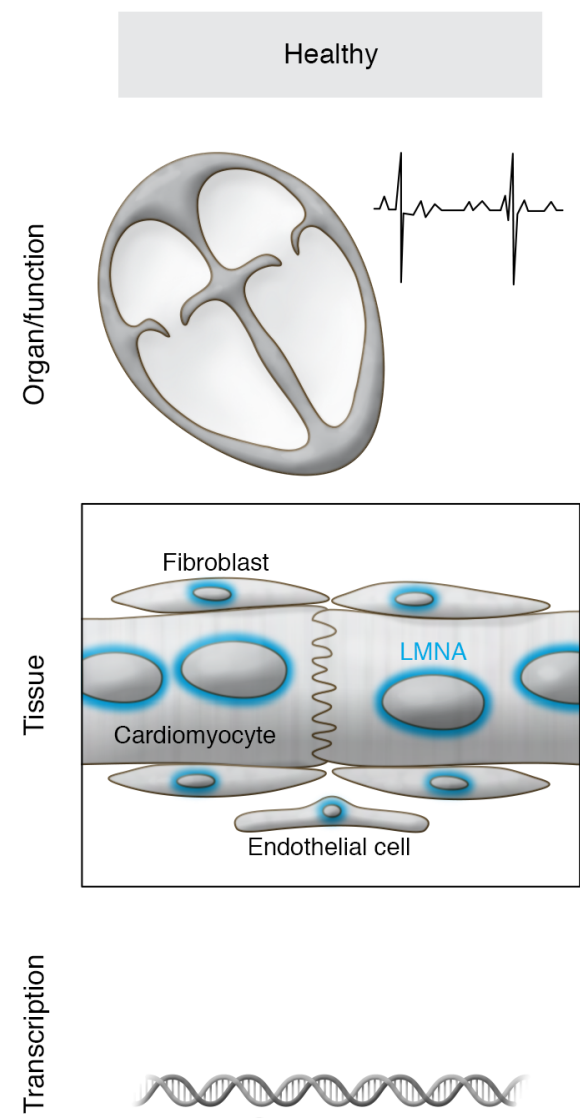
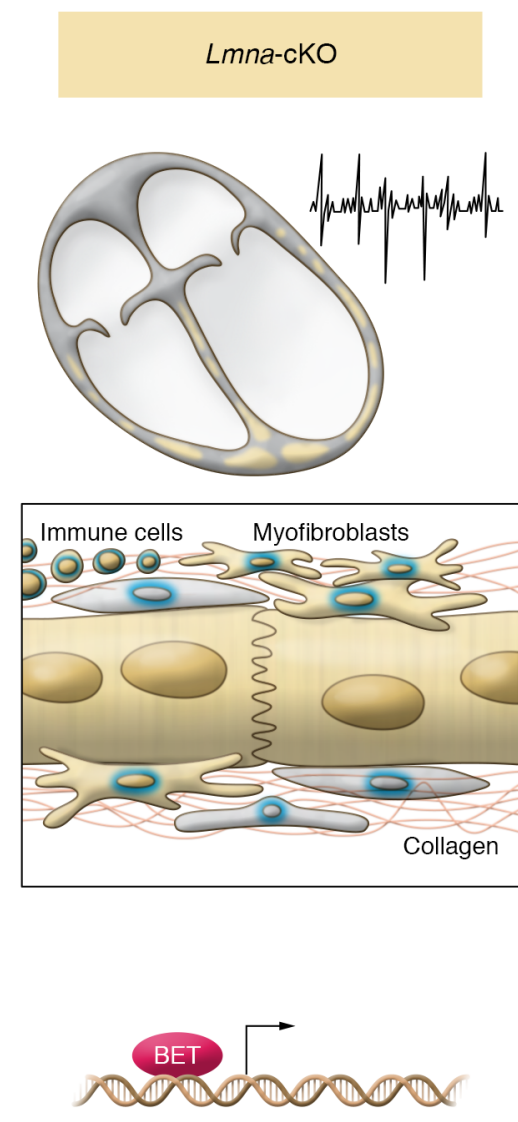
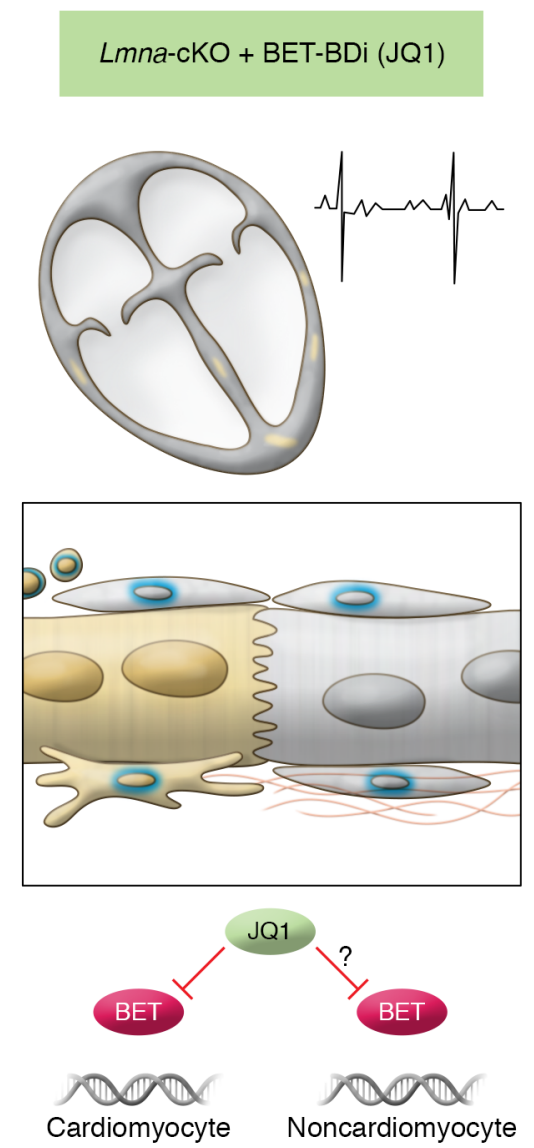

Figure 1. Cardiomyocyte-specific Lmna deletion triggers hallmark features of DCM and reveals BET proteins as druggable targets. $L m n a-c K O$ results in cardiomyocyte dysfunction, immune cell and myofibroblast activation, and collagen deposition. BET bromodomain inhibition (BET-BDi) with JQ1 improves cardiac function and attenuates induction of stress response genes. As JQ1 is administered systemically and acts upon multiple cell types simultaneously, its therapeutic effects could depend on transcription modulation of cardiomyocyte and noncardiomyocyte populations. The figure was adapted from a schematic created by Ana Catarina Silva (ana@anasilvaillustrations.com).

cifically bind the bromodomains of all four BET proteins with high affinity, reversibly displacing these coactivators from their acetylated interaction partners and inhibiting transcription of specific gene programs (4). JQ1 improves cardiac function in mouse models of pressure overload and myocardial infarction-induced $\mathrm{HF}$ (7-9), prompting Auguste and colleagues (3) to test its effects in Lmna-cKO mice. They chronically treated two-week-old mice with JQ1 and observed substantial improvements in survival and cardiac function. RNA-Seq from primary cardiomyocytes isolated from JQ1-treated mice revealed that JQ1 partially reverted the transcriptome of Lmna-deficient cardiomyocytes toward that of healthy controls. They also catalogued differentially expressed cardiomyocyte genes that were secreted factors, identifying those that could potentially crosstalk with other cell types to drive disease progression and therapeutic response to JQ1 (3).

This elegant study by Auguste and colleagues (3) highlights that cardiomyocyte-specific Lmna deletion can cause DCM, modeling several features of human cardiac laminopathy. Furthermore, the authors show that systemic administration of JQ1 can exert robust salutary effects in this model, suggesting a new therapeutic approach that targets the gene-regulatory machinery (Figure 1). Although JQ1 has previously been shown to improve heart function in mice with acquired forms of HF (7-9), the current work extends these therapeutic effects to a model of heritable human DCM. While the root cause abnormality in Lmna-cKO mice is cardiomyocyte intrinsic, secondary abnormalities in noncardiomyocytes are likely contributors to HF progression in this setting. The RNA-Seq data from isolated cardiomyo- cytes demonstrate that JQ1 has a clear beneficial effect on this cell type. However, as JQ1 is administered systemically and can act upon multiple cell types simultaneously, there may be direct effects in noncardiomyocyte populations, such as fibroblasts, immune cells, smooth muscle cells, and endothelial cells. In vitro experiments demonstrate that BRD4 plays a key role in stress-mediated activation of each of these aforementioned cell types (7, 10-13). Furthermore, in models of acquired HF, bulk RNA-Seq of heart tissue shows that JQ1 preferentially suppresses profibrotic and proinflammatory gene programs (9), although the precise cell populations mediating these effects have not been parsed in vivo. It will be critical to survey these cellular compartments using single cell-based interrogation to determine the temporal changes in cell state that occur in Lmna-cKO mice and other models of lami- 
nopathy, under both basal and JQ1-treated conditions. These unbiased analyses may also elucidate the molecular mechanisms by which ailing cardiomyocytes trigger stress responses in neighboring cells, potentially identifying new druggable factors that fuel HF progression.

Conditional genetic mouse models are complementary approaches to understand the cell-specific effects of BET proteins in vivo. Because systemic deletion of Brd4 or $\mathrm{Brd} 2$ in the mouse germline results in developmental abnormalities (14), inducible deletion in adult animals will need to be pursued for most cell types. While conditional genetic approaches can annotate allele-specific and cell type-specific effects, we emphasize that gene deletion is an entirely different molecular perturbation than small-molecule BET bromodomain inhibition. First, BET bromodomain inhibitors like JQ1 simultaneously bind to BRD2, -3 , and -4 in an equipotent manner (4). Second, JQ1 does not reduce BET protein abundance but instead competitively binds their acetyl-lysine recognition pockets and displaces them from their acetylated binding partners. Finally, JQ1 is dose titratable and reversible, allowing for partial and transient inhibitory effects in vivo, something that simply cannot be achieved by gene deletion. A clear difference between these approaches is highlighted by the observation that $\mathrm{Brd} 4$ deletion in adult mouse cardiomyocytes leads to decreased metabolic gene expression and progressive HF (15). In contrast, Auguste and colleagues find that JQ1 exerts beneficial effects on cardiomyocytes in their model of Lmna deficiency (3). To date, efforts to develop small molecules that specifically bind individual BET family members have been unsuccessful, given the high structural homology between the bromodomains of BRD2, BRD3, and $\mathrm{BRD} 4$. A deeper understanding of the protein interactomes and chromatin localization of each of these proteins in relevant cell types may facilitate the development of more specific inhibitors, potentially via altering non-bromodomain-dependent interactions. Overall, we emphasize that comparisons between genetic and chemical biological approaches for BET inhibition need to be interpreted with caution. We posit that the potent salutary effects of chemical BET bromodomain inhibitors in HF are actually due to their simultaneous binding of BRD2, BRD3, and BRD4 in multiple cell types, a perturbation that is very difficult to model using gene deletion approaches.

\section{Possible mechanistic links between lamins and BETs}

Another fascinating question Auguste et al. (3) raise is whether there is a specific mechanistic link between lamin $\mathrm{A}$ and BRD4. The nuclear lamina can regulate large-scale chromatin organization and gene expression via physically tethering large regions of the genome, called lamina-associated domains (LADs), and maintaining them in a transcriptionally inactive state (16). One intriguing hypothesis is that mutations or deficiency in LMNA causes inappropriate untethering of LADs from the nuclear periphery, leading to aberrant chromatin activation, recruitment of BRD4, and gene transcription. It will be important to assess whether BET proteins can form complexes with components of the nuclear lamina and directly participate in LAD regulation. An alternative and more likely possibility is that BET protein activation is an indirect consequence of cardiomyocyte stress. Lmna deficiency may initially perturb basal cardiomyocyte homeostasis, leading to secondary triggering of stress signaling cascades, which stimulate stress-activated transcription factors and recruitment of BET proteins to regulatory DNA. This indirect mechanism can occur in cardiomyocytes but can also mediate robust transcriptional changes in other cell types, although the precise mechanisms underlying such cellular crosstalk remain poorly understood.

\section{Translational potential for human $\mathrm{HF}$}

Can BET bromodomain inhibition ever be a viable therapy for human HF? Observations in preclinical models of acquired and heritable HF support that BET bromodomain inhibition can have broad therapeutic benefit $(3,7-9,17)$, possibly via modulating gene expression in multiple cardiac cellular compartments. While BET bromodomain inhibitors are currently being tested in cancer clinical trials (18), their ontarget toxicities in extracardiac tissues may not permit a tractable therapeutic index for chronic disease applications, such as HF.
More tissue-restricted modulation of BET proteins will likely be required to widen this therapeutic index. Understanding the precise cell- and gene-specific functions of these proteins is a smart wager that will boost the odds of getting BET bromodomain inhibitors to cover the spread from cancer to human HF.

\section{Acknowledgments}

MA was supported by the Swiss National Science Foundation (P400PM_186704) and NIH R01 HL127240. We thank Ana Catarina Silva (ana@anasilvaillustrations.com) for permission to adapt the schematic figure.

Address correspondence to: Saptarsi M. Haldar, Amgen Research, 1120 Veterans Boulevard, South San Francisco, California 94080, USA. Phone: 650.244.3794; Email:shalda01@amgen.com.

1. Chen SN, Sbaizero O, Taylor MRG, Mestroni L. Lamin A/C cardiomyopathy: implications for treatment. Curr Cardiol Rep. 2019;21(12):160.

2. Mazzarotto F, et al. Reevaluating the genetic contribution of monogenic dilated cardiomyopathy. Circulation. 2020;141(5):387-398.

3. Auguste G, et al. BET bromodomain inhibition attenuates cardiac phenotype in myocytespecific lamin A/C-deficient mice. J Clin Invest. 2020;130(9):4740-4758.

4. Filippakopoulos P, et al. Selective inhibition of BET bromodomains. Nature. 2010;468(7327):1067-1073.

5. Alexanian M, Padmanabhan A, McKinsey TA, Haldar SM. Epigenetic therapies in heart failure. JMol Cell Cardiol. 2019;130:197-204.

6. Nicodeme E, et al. Suppression of inflammation by a synthetic histone mimic. Nature. 2010;468(7327):1119-1123.

7. Anand P, et al. BET bromodomains mediate transcriptional pause release in heart failure. Cell. 2013;154(3):569-582.

8. Spiltoir JI, et al. BET acetyl-lysine binding proteins control pathological cardiac hypertrophy. J Mol Cell Cardiol. 2013;63:175-179.

9. Duan Q, et al. BET bromodomain inhibition suppresses innate inflammatory and profibrotic transcriptional networks in heart failure. $\mathrm{Sci}$ Transl Med. 2017;9(390):eaah5084.

10. Brown JD, et al. NF- $\kappa \mathrm{B}$ directs dynamic super enhancer formation in inflammation and atherogenesis. Mol Cell. 2014;56(2):219-231.

11. Perry MM, Durham AL, Austin PJ, Adcock IM, Chung KF. BET bromodomains regulate transforming growth factor- $\beta$-induced proliferation and cytokine release in asthmatic airway smooth muscle. J Biol Chem. 2015;290(14):9111-9121.

12. Bao Y, et al. Brd 4 modulates the innate immune response through Mnk2-eIF4E pathway-dependent translational control of I $\mathrm{B} \alpha$. Proc Natl Acad Sci U S A. 2017;114(20):E3993-E4001. 
13. Stratton MS, et al. Dynamic chromatin targeting of BRD4 stimulates cardiac fibroblast activation. Circ Res. 2019;125(7):662-677.

14. Houzelstein D, Bullock SL, Lynch DE, Grigorieva EF, Wilson VA, Beddington RS. Growth and early postimplantation defects in mice deficient for the bromodomain-containing protein Brd4. Mol Cell Biol. 2002;22(11):3794-3802.
15. Padmanabhan A, et al. BRD4 interacts with GATA4 to govern mitochondrial homeostasis in adult cardiomyocytes. Posted on bioRxiv April 17, 2020. https://doi. org/10.1101/2020.04.16.041806.

16. Poleshko A, et al. Genome-nuclear lamina interactions regulate cardiac stem cell lineage restric tion. Cell. 2017;171(3):573-587.e14.
17. Antolic A, et al. BET bromodomain proteins regulate transcriptional reprogramming in genetic dilated cardiomyopathy. JCI Insight. 2020;5(15):138687.

18. Bechter O, Schöffski P. Make your best BET: The emerging role of BET inhibitor treatment in malignant tumors. Pharmacol Ther. 2020;208:107479. 